Int. J. Electrochem. Sci., 1(2006)32-46

International Journal of

ELECTROCHEMICAL

SCIENCE

www.electrochemsci.org

\title{
Ionic Conductivity of Poly(ethylene oxide) (PEO)- Montmorillonite (MMT) Nanocomposites Prepared by Intercalation from Aqueous Medium
}

\author{
C. H. Manoratne ${ }^{1}$, R. M. G. Rajapakse ${ }^{1 *}$, M. A. K. L. Dissanayake ${ }^{2}$ \\ ${ }^{1}$ Department of Chemistry, Faculty of Science University of Peradeniya, Peradeniya, Sri Lanka. \\ ${ }^{2}$ Director, Post-graduate Institute of Science, University of Peradeniya, Peradeniya, Sri Lanka. \\ *E-mail: $\underline{\text { rmgr@pdn.ac.lk }}$
}

Received: 20 March 2006 / Accepted: 15 April 2006 / Published: 25 April 2006

Solid electrolyte systems, especially solid polymer electrolyte systems have been widely used as potential technological applications in secondary energy sources and electrochromic devise nowadays. The ion-transport behavior of these systems is to be well understood to enhance electronic properties. It is apparent that these materials show ionic conductivity when ionic salts are dissolved in them. The mechanism of ionic mobility is still controversial. We have made an attempt to address these problems by choosing montmorillonite/poly(ethylene oxide)(PEO)/cation as a model system and systematically understanding the bonding, intercalation and ionic conductivity by Fourier Transformed Infrared Spectroscopy (FTIR), X-ray Diffraction (XRD) and AC-Impedance Spectroscopy respectively. The systems of $\mathrm{Na}^{+}$-montmorillonite, $\mathrm{Li}^{+}$-montmorillonite, and $\mathrm{Li}^{+}$-montmorillonite-PEO are characterized. The XRD data showed that the $\mathrm{Na}^{+}$-montmorillonite has a d-spacing of $15.49 \AA$ attributing to the presence of $\mathrm{Na}^{+}$and two water layers within interlayers of MMT at room temperature. The removal of water brings the d-spacing to $9.97 \AA$. Solution intercalation of PEO into $\mathrm{Li}^{+}$-montmorillonite results in the enhancement of $d$-spacing up to $18.73 \AA$ which readily takes up water, enhancing the $d$-spacing to $23.33 \AA$. These results show clearly that PEO is readily intercalated in montmorillonite at a composition of $6 \mathrm{wt} \%$ PEO, and leaching of PEO at successive washing takes place below $6 \mathrm{wt} \%$ of PEO. In the FT-IR spectrum of montmorillonite two types of $v(\mathrm{OH})$ are seen; isolated $v(\mathrm{OH})$ groups and those involved in hydrogen bonding. The $\mathrm{Li}^{+}$exchange decreases the extent of isolated hydroxyl groups, but the extent of $\mathrm{H}$-bonding increases. This suggests that $\mathrm{Li}^{+}$is coordinated by the octahedral unit of the clay, through the $\mathrm{O}$ atoms (ions) of free Al-O-H groups. The ionic conductivity figures measured using AC-impedance analyzer is in agree with this bonding arrangement.

It has been found that the intercalation of PEO in $\mathrm{Li}^{+}$-montmorillonite reverses the above process by the coordination of $\mathrm{Li}^{+}$ions with $\mathrm{O}$ atoms of PEO. The amount of free $\mathrm{O}-\mathrm{H}$ then increases and the extent of hydrogen bonded $\mathrm{O}-\mathrm{H}$ decreases.

Keywords: montmorillonite-polyethylene oxide nanocomposites, intercalation 


\section{INTRODUCTION}

The inevitable studies of Wright and Armand on ionically conducting polymers, called "polymer electrolytes" in the late 1970s have opened an innovative area of materials research with potential applications in the power industry. The main applications of these polymer electrolytes were in rechargeable lithium batteries as an alternative to liquid solution electrolytes [1,2]. The inventions of conducting polymers together with the extensive studies on intercalation chemistry helped to understand the microscopic structure-property relationship of nanocomposite materials based on polymer-layered silicate in the development of nanotechnology [3]. These hybrid materials with features on the scale of nanometers exhibit characteristics properties superior to conventional composites [4]. The conductivity enhancement of cation exchanged montmorillonite have been reported afore by many researchers, using clay samples from different origins [5,6]. Six typical montmorillonite clays from different locations in China have shown that the ionic conductivity of smectite intercalation compounds (SIC) depends on the original structure and compositions of smectite minerals. Samples with high cation exchange capacity (CEC), and less substitution has higher ionic conductivity and vise versa, in addition, the conductance increases with the migration of ions in the interlayer galleries $[7,8]$. The relative humidity also plays a significant role on the conductivity of the modified montmorillonite, other than the location of the montmorillonite. The effect of relative humidity on the conductivity of sodium (Na-montmorillonite) and lithium-montmorillonite (Limontmorillonite) have been reported, where similar values of increased conductivity have been observed for both samples at higher humidity, whereas at low humidity zone the Li-montmorillonite has exhibited higher conductivity than that of Na-montmorillonite [9]. The inventions of two families of fast ion conductors; a series of complex compounds based on silver iodide and $\beta$-alumina, and montmorillonite with the support of latest sophisticated physical techniques such as X-ray, neutron scattering and NMR provided considerable development in the fast ion conductors. The X-ray diffraction, $\mathrm{X}$-ray fluorescence, the ionic conductivity and electronic conductivity measurements were observed for $\mathrm{Li}^{+}, \mathrm{Na}^{+}, \mathrm{Mg}^{2+}, \mathrm{Ca}^{2+}$ and $\mathrm{Zn}^{2+}$-montmorillonite composites respectively. However, in this finding the influence of the relative humidity or the water content on conductivity of cations intercalated montmorillonite showed remarkable changes as compared to previous investigation $[10,11]$. The composite mixtures of alkali salts/MMT ( NaCl, NaI, NaSCN and LiI/MMT) have shown an enhancement in solid-state ionic conductivity than the two constituents. The mixing of different alkali salts mentioned above has been carried out by solution intercalation method and powder mixing method, and their conductivities have been measured by ac impedance technique in the temperature region of RT to $400{ }^{\circ} \mathrm{C}$ in a dry atmosphere. The ac conductivity $\mathrm{Na}^{+}-\mathrm{Or} \mathrm{Li}^{+}$-montmorillonite has shown low conductivity of $10^{-7}$ to $10^{-6} \mathrm{~S} \mathrm{~cm}^{-1}$ at $200{ }^{\circ} \mathrm{C}$, and the NaSCN-montmorillonite has shown the highest conductivity of $10^{-4} \mathrm{~S} \mathrm{~cm}^{-1}$ at $200^{\circ} \mathrm{C}$ [9].

It is apparent in the literature that the researches have not been confined only to the studying of montmorillonite based nanocomposites or conductivity but extended to cover certain other applications. One such contribution is the formation of well-dispersed metal oxides over montmorillonite matrix, which shows enhanced reactivity with gaseous reactants. Increased absorption of $\mathrm{H}_{2} \mathrm{~S}$ by $\mathrm{ZnO}$ supported on montmorillonite matrix can be cited as an example [12]. In 
addition to the widespread researches, which are being carried out to understand the structure-property of modified montmorillonite, the cation diffusion coefficient studies have given a light in elucidation of conductivity mechanism in montmorillonite. The diffusion coefficients of $\mathrm{Li}^{+}$and $\mathrm{Na}^{+}$intercalated montmorillonite have been measured by potentiostatic and galvanostatic step techniques. The investigation has showed comparatively that the presence of interlayer water molecules or other polar molecules always enhances the ionic conductivity and diffusion coefficient. Moreover, the diffusion coefficients of MMTLi and MMTNa are proportional to the water content less than 20\% [13]. In addition to diverse researches of the properties of layered minerals, the transport properties in layered Vermiculite have also gained a great interest in many researchers. In these studies, the ionic conductivity of magnesium and calcium ion-exchanged Vermiculite has been reported from $5{ }^{\circ} \mathrm{C}$ to 90 ${ }^{\circ} \mathrm{C}$. They have comparatively presented from the results of sodium conductivity data with tracer sodium and proton NMR studies that the dominant migrating species in Vermiculite are counter ions and not protons [14]. It is not much attributed fact, that in the literature of the mixtures of two model clays. However, an investigation of physical property tests of a mixture of kaolinite and montmorillonite has filled this vacuum. It has shown that these properties are influenced by exchangeable cations ( $\mathrm{Ca}, \mathrm{Na})$ and specific surface $(\mathrm{S})[15]$.

In this publication, we describe the preparation of MMT-PEO nanocomposites via aqueous solution phase intercatation of PEO within the intergalleries of MMT, their characterization and ionic conductivity studies.

\section{EXPERIMENTAL PART}

\subsection{Materials}

The clay, sodium montmorillonite (Bentonite) was purchased from (Aldrich Chemicals, Milwaukee, WI). The crystalline poly(ethylene oxide) (PEO) (Aldrich Chemicals) with a weight-average molecular weight of 400,000, and lithium chloride ( $\mathrm{LiCl}$ ) (Hopkin \& Williams, G. P. R), were used as received. Generally, the PEO, and salts are highly hygroscopic, therefore it is required to ensure the dryness of these materials prior to sample preparation. Normally, the salt is dried at $80-150{ }^{\circ} \mathrm{C}$ for $24 \mathrm{~h}$ and then at room temperature for $24 \mathrm{~h}$ under dynamic partial vacuum $\left(10^{-5} \mathrm{~Pa}\right)$. The PEO is dried at $40^{\circ} \mathrm{C}$ for 24 $\mathrm{h}$ under high vacuum.

\subsection{Methods}

The clay sample (montmorillonite) may consist of organic and inorganic impurities Therefore, it is desirable to eliminate them as much as possible. The centrifuging and washing method were employed, which will have a least effect to the structure and chemical composition. The montmorillonite $(50 \mathrm{~g}$ ) was suspended in excess de-ionized water and stirred magnetically for about 48 $\mathrm{h}$ at room temperature. The suspension was centrifuged at $5000 \mathrm{rpm}$ for two minutes, and the clear supernatant was decanted. The resultant slurry was re-dispersed in excess of de-ionized water and the above procedure continued. This procedure was repeated several times until the supernatant was free 
of any impurity. The clear supernatant observed, confirmed the absence of organic impurities in the montmorillonite. This slurry thus obtained kept in the normal environment to air-drying and used in other applications.

The $\mathrm{Li}^{+}$ion intercalation has been carried out by suspending $15.0 \mathrm{~g}$ of purified montmorillonite slurry in $200 \mathrm{~cm}^{3}$ of $1.0 \mathrm{~mol} \mathrm{dm}^{-3} \mathrm{LiCl}(\mathrm{aq})$ solution. The suspension was then stirred for $48 \mathrm{~h}$ continuously. The resultant mixture was then centrifuged at $5000 \mathrm{rpm}$ for two minutes, and the supernatant was decanted. This was followed by a series of washings with de-ionized water and centrifugation until complete removal of chloride ions in supernatant was achieved [tested using $\mathrm{AgNO}_{3}(\mathrm{aq})$ ]. The slurry thus obtained is MMTLi.

$15.0 \mathrm{~g}$ of MMTLi was suspended in $200 \mathrm{ml}$ of de-ionized water and stirred for few minutes and then added 1.0 of PEO into that solution. The suspension was stirred for $48 \mathrm{~h}$ continuously until a homogeneous mixture was obtained. The suspension was centrifuged and supernatant was decanted. This procedure was carried out several times. The slurry thus obtained is the MMTLiPEO.

Another $15.0 \mathrm{~g}$ of purified montmorillonite was suspended in $200 \mathrm{~cm}^{3}$ de-ionized water and stirred for few minutes, and then $1.0 \mathrm{~g}$ of PEO was added and allowed to stir for $48 \mathrm{~h}$ continuously. The suspension was centrifuged and washed as mentioned above. The slurry thus obtained is the MMTPEO.

\subsection{Instrumentation and Characterization}

The ac impedance measurements of the MMT and it's intercalated systems were carried out using a computer-interfaced SI 1260 IMPEDANCE/GAIN-PHASE ANALYSER over the frequency range of 8-10 MHz at the voltage of $20 \mathrm{mV}$. The samples were sandwiched between two stainless steel blocking electrodes $\left(1.27 \mathrm{~cm}\right.$ diameter.) and the temperature changed from $25^{\circ} \mathrm{C}$ to $80{ }^{\circ} \mathrm{C}$. At each temperature the conductivity value was calculated using the resistance value from the real axis intercept of the semicircle in the complex impedance plane plot and the sample dimensions.

In this work the X-ray diffraction patterns were conducted by SIEMENCE X-ray diffractometer (SIEMENCE, German). The slurries of each of above composite were suspended in a small volume ( $5.00 \mathrm{~cm}^{3}$ ) of distilled water, and the suspension was poured onto glass plates allowing to dry in air for a few days. The samples of each of above type was X-rayed after a various treatments including airdrying, moisturizing, and heating in a furnace at $100{ }^{\circ} \mathrm{C}, 150{ }^{\circ} \mathrm{C}, 200{ }^{\circ} \mathrm{C}, 250{ }^{\circ} \mathrm{C}$ and $300{ }^{\circ} \mathrm{C}$ for 1 hour.

FTIR spectra were collected on a JASCO FT/IR-410 spectrometer from 400 to $4000 \mathrm{~cm}^{-1}$. The samples were prepared by mixing the well-dried sample with dry $\mathrm{KBr}$ powder, having the ratio 1:13. The mixture was pressed into a disc of $13 \mathrm{~mm}$ in diameter and thick $<1 \mathrm{~mm}$. Prior to the analysis of FTIR each pellet was kept in the desiccator for several days to avoid the disturbances from water molecules. 


\section{RESULTS AND DISCUSSION}

\subsection{XRD Studies}

Figure 1 (a), (b), and (c) show the XRD spectra of natural montmorillonite, kept in ambient laboratory environment, after heat-treating at $150{ }^{\circ} \mathrm{C}$ for $2 \mathrm{hrs}$, and moisturising with water respectively. In MMT the layers are stacked along a line perpendicular to (001) basal plane. Generally, the interlayer of pure MMT contains two planes of water molecules compensated with inter gallery exchangeable cations. These interlayer water molecules are loosely bound by weak van der Walls forces as such they can be removed easily at higher temperature. When the same samples were oven dried at $150{ }^{\circ} \mathrm{C}$ for 2 hours, the (001) layer spacings reduced to $9.96 \AA$, which attributed to the absence of water molecules within the MMT, however the layer spacing increases up to $13 \AA$ when the cations are surrounded by one water-layer [16]. The layer spacing increases up to $18.9 \AA$ when the surface of pure MMT was wet with moisture. This indicates that the interlayers contain three water layers in this condition $[17,18]$. Hence, it can be concluded that the montmorillonite samples used in the present work with d-spacing of around $10 \AA$ contain fully dehydrated cations, samples with d-spacings of $13.0 \AA$ contained a single water layer, samples with d-spacings of $15 \AA$ contained two water layers, and samples with d-spacings of $18.0 \AA$ contained three water layers within interlayer spaces.

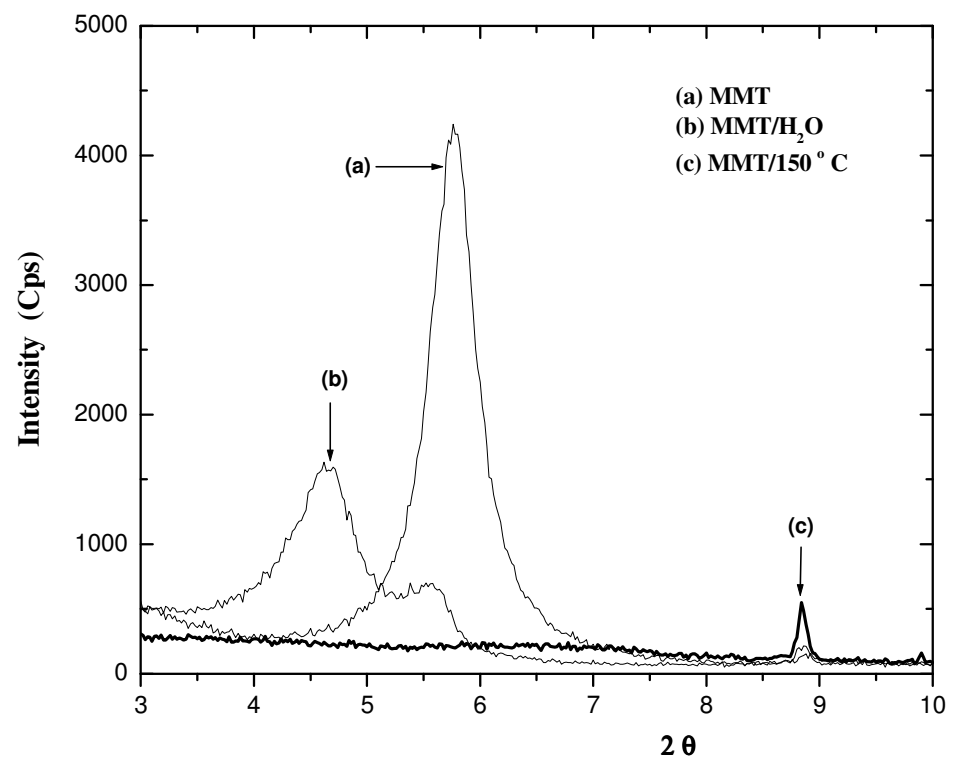

Figure 1: The d-spacing profile for (a) pure montmorillonite (MMT), (b) with moisturizing $\left(\mathrm{MMT} / \mathrm{H}_{2} \mathrm{O}\right)$ and $(\mathrm{c})$ heated at $150{ }^{\circ} \mathrm{C}$ for $2 \mathrm{~h}\left(\mathrm{MMT} / 150{ }^{\circ} \mathrm{C}\right)$

The $\mathrm{d}$ value of pure montmorillonite will not with the intercalation of $\mathrm{Li}^{+}$cations, and it seems remained at $15.57 \AA$ almost unchanged. The hydration power of $\mathrm{Li}^{+}$cations are high, therefore it can be assumed that the $\mathrm{Li}^{+}$cations present in the interlayer gallery would be hydreated, in addition to the weakly bonded water molecules within the layers. The intercalation of $\mathrm{Li}^{+}$ions within the interlayer spacing of MMT has a significant effect on water uptake that the MMT becomes hydrophobic, see 
figure 2. The speculation is that $\mathrm{Li}^{+}$ions migrate into the octahedral sheet and neutralize the layer charge if the charge is due to octahedral substitution. The elimination of charge converts MMT to a pyrophyllite-like mineral that does not expand upon treatment with water, glycerol, or ethylene glycol [17]. However, at higher temperatures the water gets removed as that of pure MMT.

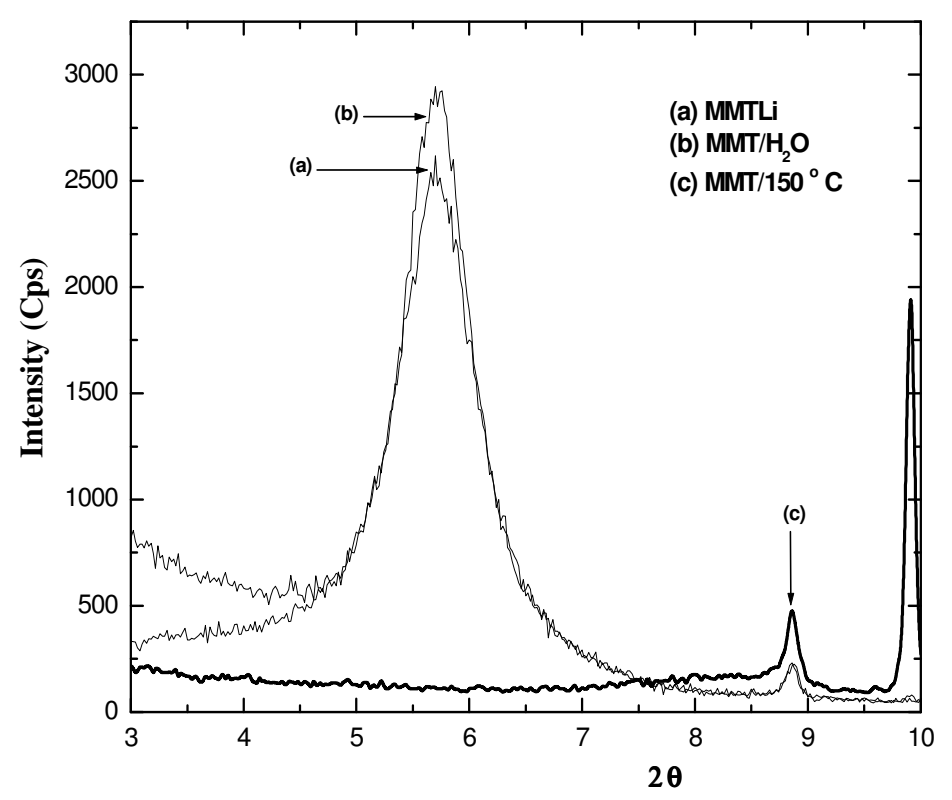

Figure. 2: The d-spacing profile for (a) $\mathrm{Li}^{+}$cations intercalated montmorillonite (MMTLi), (b) with moisturizing (MMTLi/ $\left.\mathrm{H}_{2} \mathrm{O}\right)$ and (c) heated at $150{ }^{\circ} \mathrm{C}$ for $2 \mathrm{~h}\left(\mathrm{MMTLi} / 150{ }^{\circ} \mathrm{C}\right)$

PEO gets easily intercalated by solution intercalation method into the interlayer spacing of montmorillonite. The d-spacing observed for MMTPEO at the room temperature is $18.73 \AA$. It is assumed that PEO molecules get coordinated to interlayers perpendicular to the (001) basal plane and not to the external surface of the montmorillonite as XRD patterns revealed the expansion of interlayer spacing even with washing and centrifuging for many times. As evidenced the water molecules within the interlayer spacing of montmorillonite can be removed at higher temperature (above $120{ }^{\circ} \mathrm{C}$ ). The MMTPEO gave a $\mathrm{d}$ value of $17.17 \AA$ at $150^{\circ} \mathrm{C}$. This implies that the interlayer spacing are occupied all most all by the PEO molecules. The d-spacing further increases readily to a value of $23.33 \AA$ when moisturizing the MMTPEO samples (see fig. 3). We have observed that the intercalation of PEO can be done at $6 \mathrm{wt} \%$ PEO with water as the solvent that has not been reported previously elsewhere.

It has been reported previously that $\mathrm{Li}^{+}$cations intercalated montmorillonite shows some reluctance to up take water or glycerol, or ethylene glycol within the interlayer gallery. However, in contrast to that the MMTLi shows great tendency to intercalate PEO within the interlayers. But in this system it was observed that the d-spacings of the respective conditions such as at room temperature and with moisturizing show slightly lower value than those in the case of MMTPEO. The d-spacing of MMTLiPEO at room temperature is about $18.66 \AA$, with the moisturizing it increases to the value of 
about $22.62 \AA$ (see fig. 4). This may have been due to the fact that some regions within the interlayer spacings have been occupied by hydrated $\mathrm{Li}^{+}$cations therefore now the rooms that were available in pure montmorillonite became less. Similar to the MMTPEO system this system also shows expanded $\mathrm{d}$-spacing at higher temperatures. As observed the d-spacing at $150{ }^{\circ} \mathrm{C}$ is about $17.08 \AA$. This confirms that PEO molecules are bonded to the layers of MMT, and $\mathrm{Li}^{+}$cations, and not to the external surface of the clay particles. The d-spacing values of MMT, MMTLi, MMTLiPEO, and MMTPEO determined at room temperature $\left(\mathrm{RT} /{ }^{\circ} \mathrm{C}\right), 100{ }^{\circ} \mathrm{C}, 150{ }^{\circ} \mathrm{C}, 200{ }^{\circ} \mathrm{C}, 250{ }^{\circ} \mathrm{C}$, and $300{ }^{\circ} \mathrm{C}$ are summarized in table 1. The d-spacing temperature profiles of each of above system are shown in fig. 8 to have a better comparison.

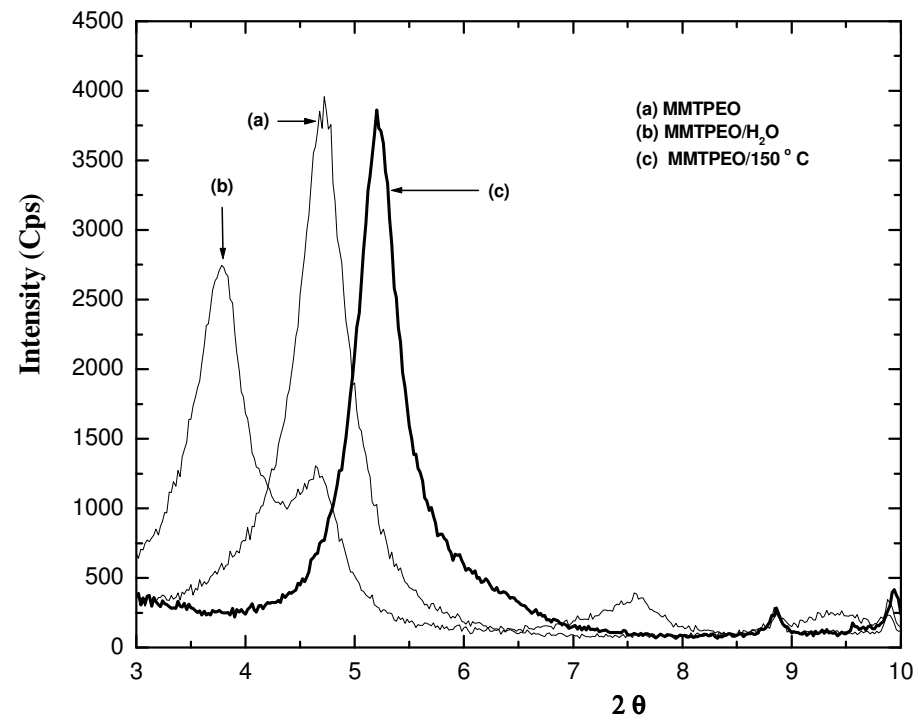

Figure 3: The d-spacing profile for (a) PEO intercalated montmorillonite (MMTPEO), (b) with moisturizing (MMTPEO/ $\left.\mathrm{H}_{2} \mathrm{O}\right)$ and (c) heated at $150{ }^{\circ} \mathrm{C}$ for $2 \mathrm{~h}\left(\mathrm{MMT} / 150{ }^{\circ} \mathrm{C}\right)$

Table 1: The d-spacing of the Na-montmorillonite, MMTLi, MMTPEO, and MMTLiPEO at RT (26 $\left.{ }^{\circ} \mathrm{C}\right), 100{ }^{\circ} \mathrm{C}, 150{ }^{\circ} \mathrm{C}, 200{ }^{\circ} \mathrm{C}, 250{ }^{\circ} \mathrm{C}$ and $300{ }^{\circ} \mathrm{C}$

\begin{tabular}{|l|c|c|c|c|c|c|c|}
\hline \multirow{2}{*}{ Sample Name } & \multicolumn{7}{|l|}{ Change of inter gallery spacing $\mathbf{d} / \mathbf{A}$} \\
\cline { 2 - 9 } & $\mathbf{\mathbf { R T } ^ { \circ } \mathbf { C }}$ & $\begin{array}{l}\text { Addition of } \\
\text { water at } \mathbf{R T} \\
\left(\mathbf{2 6}{ }^{\circ} \mathbf{C}\right)\end{array}$ & $\mathbf{1 0 0}^{\circ} \mathbf{C}$ & $\mathbf{1 5 0}^{\circ} \mathbf{C}$ & $\mathbf{2 0 0}^{\circ} \mathbf{C}$ & $\begin{array}{c}\mathbf{2 5 0} \\
{ }^{\circ} \mathbf{C}\end{array}$ & $\begin{array}{c}\mathbf{3 0 0} \\
{ }^{\circ} \mathbf{C}\end{array}$ \\
\hline Montmorillonite & 15.19 & 18.97 & 14.85 & 9.96 & 9.97 & 9.58 & 9.96 \\
\hline BLi & 15.57 & 15.68 & 15.27 & 9.97 & 9.95 & 9.97 & 9.97 \\
\hline BPEO & 18.73 & 23.33 & 18.55 & 17.17 & 17.09 & 13.52 & 14.49 \\
\hline BLiPEO & 18.66 & 22.62 & 18.16 & 17.08 & 17.40 & 13.45 & 13.38 \\
\hline
\end{tabular}




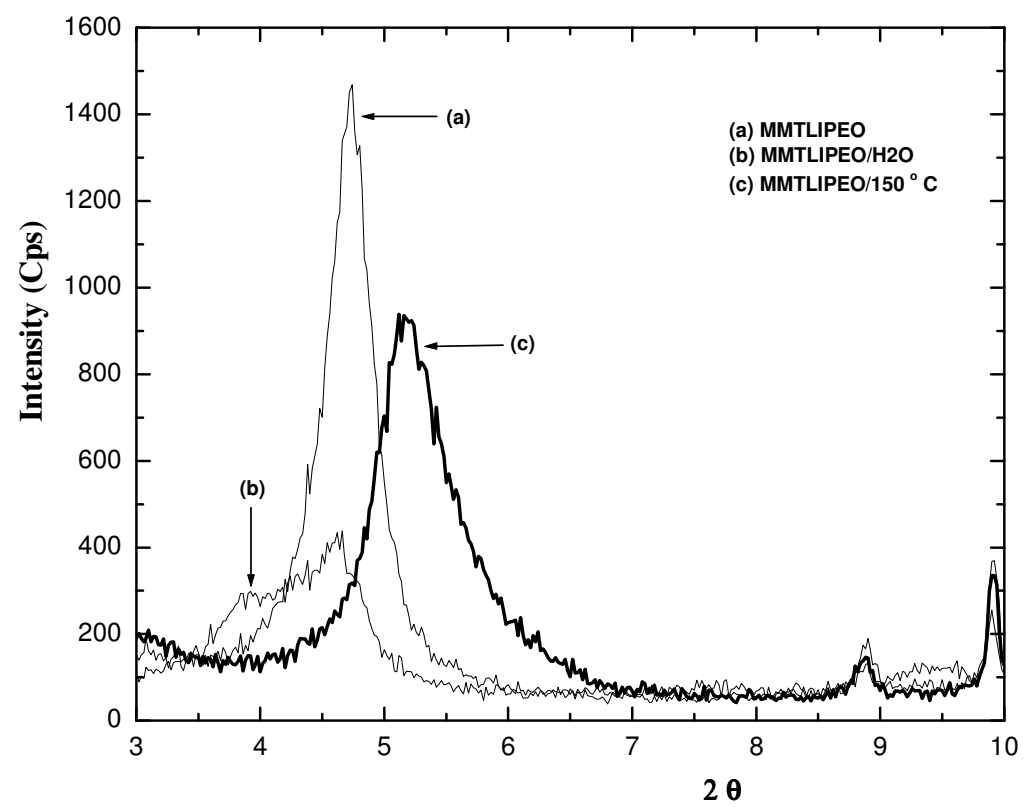

Figure 4: The d-spacing profile for (a) $\mathrm{Li}^{+}$cations intercalated within MMTPEO (MMTLiPEO), (b) with moisturizing (MMTLiPEO/ $\left.\mathrm{H}_{2} \mathrm{O}\right)$ and (c) heated at $150{ }^{\circ} \mathrm{C}$ for $2 \mathrm{~h}\left(\mathrm{MMTLIPEO} / 150{ }^{\circ} \mathrm{C}\right)$

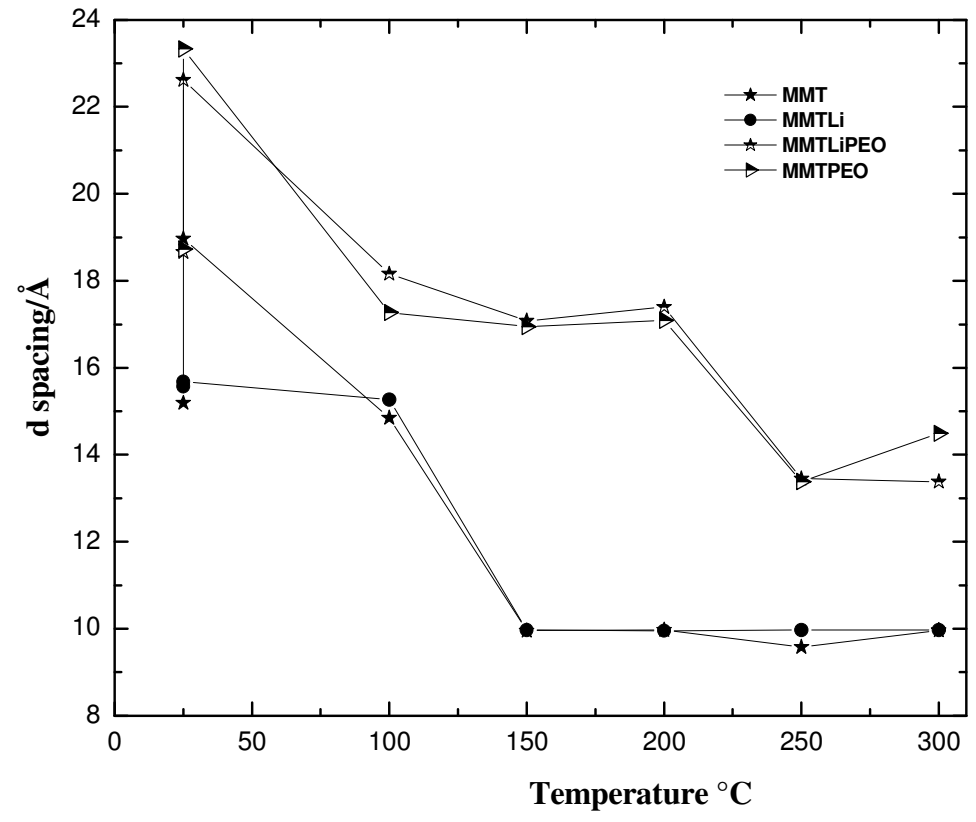

Figure 5: The d-spacing temperature profiles for MMT, MMTLi, MMTLiPEO and MMTPEO. Note that points are connected only for clarity. The lines may not show continuous variation. 


\subsection{Ionic Conductivity Studies}

The typical temperature dependent conductivity Arhenius plots measured for MMT, MMTLi, MMTPEO, and MMTLiPEO in the range of $25^{\circ} \mathrm{C}-80{ }^{\circ} \mathrm{C}$ are shown in fig. 6 . The corresponding conductivity values obtained at various temperatures are listed in table 2.

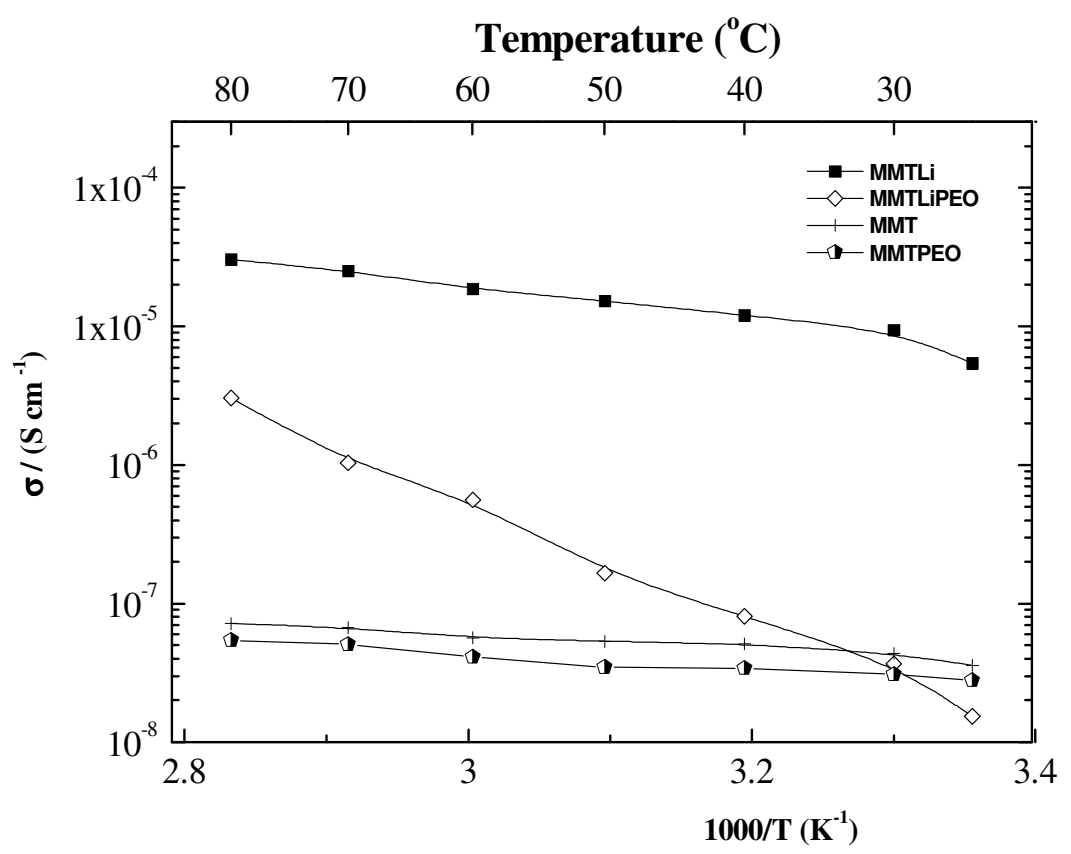

Figure 6: Temperature dependence of conductivity for MMT, MMTLi, MMTPEO, and MMTLiPEO.

Table 2: Ionic conductivity for MMT, MMTLi, MMTLiPEO, and MMTPEO at some selected temperatures.

\begin{tabular}{|l|c|c|c|c|c|c|c|}
\hline Sample & $\mathbf{2 5}^{\circ} \mathbf{C}$ & $\mathbf{3 0}^{\circ} \mathbf{C}$ & $\mathbf{4 0}^{\circ} \mathbf{C}$ & $\mathbf{5 0}^{\circ} \mathbf{C}$ & $\mathbf{6 0}^{\circ} \mathbf{C}$ & $\mathbf{7 0}^{\circ} \mathbf{C}$ & $\mathbf{8 0}^{\circ} \mathbf{C}$ \\
\hline MMT & $3.58 \mathrm{E}-8$ & $4.38 \mathrm{E}-8$ & $5.12 \mathrm{E}-8$ & $5.33 \mathrm{E}-8$ & $5.64 \mathrm{E}-8$ & $6.68 \mathrm{E}-8$ & $7.19 \mathrm{E}-8$ \\
\hline MMTLi & $5.43 \mathrm{E}-6$ & $9.33 \mathrm{E}-6$ & $1.20 \mathrm{E}-5$ & $1.52 \mathrm{E}-5$ & $1.87 \mathrm{E}-5$ & $2.50 \mathrm{E}-5$ & $3.04 \mathrm{E}-5$ \\
\hline MMTLiPEO & $1.53 \mathrm{E}-8$ & $3.69 \mathrm{E}-8$ & $8.09 \mathrm{E}-8$ & $1.66 \mathrm{E}-7$ & $5.62 \mathrm{E}-7$ & $1.03 \mathrm{E}-6$ & $3.03 \mathrm{E}-6$ \\
\hline MMTPEO & $2.80 \mathrm{E}-8$ & $3.07 \mathrm{E}-8$ & $3.40 \mathrm{E}-8$ & $3.48 \mathrm{E}-8$ & $4.13 \mathrm{E}-8$ & $5.07 \mathrm{E}-8$ & $5.41 \mathrm{E}-8$ \\
\hline
\end{tabular}

The montmorillonite used in our study showed a conductivity of $3.58 \times 10^{-8} \mathrm{~S} \mathrm{~cm}^{-1}$ at room temperature. The water content in MMT used for conductivity measurement was found to be $15 \%$, see table 3. However, the maximum conductivity arrived at higher temperature $\left(80{ }^{\circ} \mathrm{C}\right)$ is around $7.19 \times$ $10^{-8} \mathrm{~S} \mathrm{~cm}^{-1}$. Prior to the ac impendence measurements we have carried out dc polarization test on each system; however, it shows that the ionic conductivity of purified montmorillonite could not be 
observed due to low sensitivity of the apparatus. But in the case of ac characterization plot small ionic conductivity can be observed. It has been reported previously that the interlayer absorbed water molecules in montmorillonite has a considerable impact on its ionic conductivity and diffusion coefficient: the higher the water content, the lower the activation energy, and greater the ionic conductivity and diffusion coefficient [13]. A hypothesis has been developed describing that the compensated cations in MMT are in thermal disturbance [11]. In addition to that a NMR study of MMT and vermiculite has shown two relaxation processes. One reveals the motion of free water and the other contributes to the hydrated $\mathrm{Na}^{+}$cations [19, 20, 21]. It was assumed from the available data that with the increase of temperature the mobility of interlayer exchangeable cations in this case $\mathrm{Na}^{+}$is enhanced. This gives rise to the conductivity increment with varying temperature in montmorillonite. This conductivity of purified MMT is much less with compare to other materials and composite systems.

Table 3: Conductivity values of montmorillonite and its derivatives in relation to the water content observed at room temperature.

\begin{tabular}{|l|l|l|}
\hline Sample name & Cond. $\left(\sigma / \mathrm{S} \mathrm{cm}^{-1}\right)$ at $298 \mathrm{~K}$ & Water content $(\%$ by mass) \\
\hline MMT & $3.58 \times 10^{-8}$ & 15 \\
\hline MMTLi & $5.43 \times 10^{-6}$ & 13 \\
\hline MMTPEO & $2.80 \times 10^{-8}$ & 3 \\
\hline MMTLiPEO & $1.53 \times 10^{-8}$ & 1.7 \\
\hline
\end{tabular}

The table 3 gives the conductivity values with their water content measured at room temperature. Our objective here is to develop a high conductivity material, which satisfies the in situ requirements enhancing the applications in opto-electronic devices and high-energy secondary batteries. It has been observed that $\mathrm{Li}^{+}$ions intercalated montmorillonite has an enhanced conductivity i.e. about $5.43 \times 10^{-6}$ $\mathrm{Scm}^{-1}$ at room temperature at $13 \%$ water content. We could observe a gradual increase of conductivity with the increases of temperature up to $80{ }^{\circ} \mathrm{C}$. The conductivity observed at $80^{\circ} \mathrm{C}$ is $3.04 \times 10^{-5} \mathrm{~S} \mathrm{~cm}^{-}$ ${ }^{1}$. In this case the mobility of $\mathrm{Li}^{+}$increases as the temperature increases, and it can hop through water network. Samples with high cation exchange capacity (CEC), and less substitution has higher ionic conductivity and vise versa, in addition, the conductance increases with the migration of ions in the interlayer galleries [22,23]. The MMT used in this study has the cation exchange capacity of 100 meq/100 g. As confirmed by the XRD studies the MMTLi has the hydrophobic nature, therefore water absorption could be less. As shown in the table 3, the water content in MMTLi is reduced with compare to the pure MMT. Therefore, the protonic conductivity due to the presence of water molecules could be minimized. The overall results thus showed that the conductivity of MMTLi is mainly due to the $\mathrm{Li}^{+}$cation than water molecules.

Due to the screening effect of the interlayer bonded water, the Coulombic attraction of the layers to the exchanged cation becomes weaker $[11,13]$. Therefore, the activation energy of $\mathrm{Li}^{+}$cations decreases. This phenomenon also contributes to the increase of ionic conductivity with the increase of 
temperature. The MMTPEO showed a lower conductivity at room temperature. The increase of conductivity with the temperature increase can be seen parallel to that of MMT, but this lies below the MMT behavior. This can be attributed that the PEO molecules arranges in a disordered manner within the clay structure restricting the free hydroxyl groups and coordinates with interlayer cations making a net structure [3]. The change of asymmetric $\mathrm{CH}_{2}$ stretching and bending vibrations confirmed this phenomenon. The XRD data showed that the interlayers of MMT are mainly occupied by PEO molecules, thus reducing interlayer water content. The water content at the conductivity measurement was found to be $3 \%$. This causes the lowering of both protonic conductivity and mobility of interlayer exchangeable cations. In the system of MMTLiPEO the conductivity behavior is somewhat different. At room temperature the ionic conductivity is reduced to $1.53 \times 10^{-8} \mathrm{~S} \mathrm{~cm}^{-1}$. But it increases rapidly with the increase of temperature and reached to a value of $3.03 \times 10^{-6} \mathrm{~S} \mathrm{~cm}^{-1}$. The intercalation of PEO within MMTLi prefers the interaction of $\mathrm{Li}^{+}$cations with ether oxygen of PEO. Thus it reduces the mobility of $\mathrm{Li}^{+}$cations. Similar to MMTPEO system the interlayers in MMTLiPEO are occupied by PEO molecules, forming a net like structure within the clay layers. The water content at the conductivity measurement was found to be $1.7 \%$. In addition to that PEO within the montmorillonite increases the amount of free $\mathrm{O}-\mathrm{H}$, thus reducing the extent of hydrogen bonded $\mathrm{O}-\mathrm{H}$. Therefore the conductivity at room temperature is reduced. However, the increase of temperature enhances the $\mathrm{Li}^{+}$ mobility favorably. As such now the conductivity increases as the increase of temperature.

\subsection{FT-IR studies}

Figure 7 shows the FTIR spectra obtained for MMT, MMTLi, MMTPEO, and MMTLiPEO in the wavenumber range 400 to $4000 \mathrm{~cm}^{-1}$. In pure MMT a clear broad band in the region $3300-3700 \mathrm{~cm}^{-1}$ was observed which, corresponds to the $-\mathrm{OH}$ stretching $(\mathrm{v}(-\mathrm{OH}))$ vibration. The band has two features at $3624 \mathrm{~cm}^{-1}$ and at $3440 \mathrm{~cm}^{-1}$ showing in the presence of two types of $\mathrm{O}-\mathrm{H}$ groups: isolated $\mathrm{OH}$ groups and those involved in hydrogen bonding [24]. The nanocomposite prepared by intercalation of either $\mathrm{Li}^{+}$or PEO within the interlayer spacing of MMT shows strategic features in the FTIR spectra than those of pure constituents. In MMTLi system, it is clear that the intercalation of $\mathrm{Li}^{+}$cations has a significant impact upon the structure of MMT. The typical bands of $3624 \mathrm{~cm}^{-1}$ and $3440 \mathrm{~cm}^{-1}$ in MMT are transformed to a new intensed broad band centered at around $3460 \mathrm{~cm}^{-1}$ in MMTLi which corresponds to the decrease of isolated hydroxyl groups and the increase of hydrogen bonding $-\mathrm{OH}$ groups. This suggests that $\mathrm{Li}^{+}$cations interact with the $-\mathrm{OH}$ groups of the clay, through the $\mathrm{O}$ atoms (ions) of free Al-O-H groups. The peak at $1634 \mathrm{~cm}^{-1}$ corresponds to the bending vibrational mode of hydrated water molecules and weakly bonded water molecules. The corresponding band in MMTLi is intensed. It can be suggested that the interlayer $\mathrm{Li}^{+}$cations are more hydrated than pure MMT; therefore the presence of $-\mathrm{OH}$ groups is abundant. The strong interactions of MMT to the $\mathrm{Li}^{+}$cations were further confirmed, as the change of typical vibrational modes in MMT is apparent. The typical bands of $920 \mathrm{~cm}^{-1} \mathrm{v}(\mathrm{Al}-\mathrm{O}-\mathrm{H}), 796 \mathrm{~cm}^{-1} \mathrm{v}(\mathrm{Al}(\mathrm{Mg})-\mathrm{O}-\mathrm{H}), 1040-1120 \mathrm{~cm}^{-1} \mathrm{v}(\mathrm{Si}-\mathrm{O})$ and the bands

appear at $525 \mathrm{~cm}^{-1}$ and $470 \mathrm{~cm}^{-1}$ which correspond to the $v(\mathrm{Al}(\mathrm{Mg})-\mathrm{O}-\mathrm{Si})$ were observed in pure MMT [25]. However, a clear observation can be seen in MMTLi, in which the intensities of above modes were affected and reduced. This suggests that $\mathrm{Li}^{+}$cations significantly interact with $-\mathrm{OH}$ groups 
in the octahedral layer and coordination with $\mathrm{Si}-\mathrm{O}$ in tetrahedral layer of the MMT clay. The above results are consistent with the results of conductivity measurements and XRD experiments.

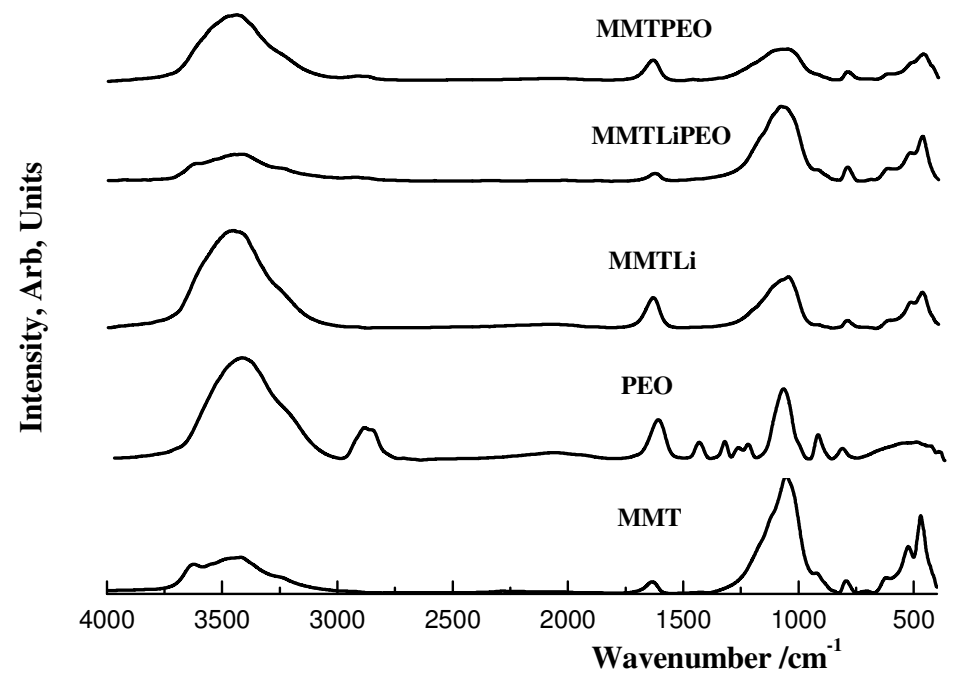

Figure 7: FTIR spectra of MMT, MMTLi, MMTPEO, MMTLiPEO, and PEO

It was observed that the intercalation of PEO within MMT shows the similar type of effect on MMT structure and in return on PEO crystallinity. The band at $3624 \mathrm{~cm}^{-1}$ is absent in the MMTPEO. This is due to the interaction between the ether-oxygen of PEO segments and the free -OH groups of clay layers via hydrogen bonding [26]. This can be seen as the disappearing of the band at $3624 \mathrm{~cm}^{-1}$ and appearing of well-transformed peak at $3447 \mathrm{~cm}^{-1}$. In the pure PEO spectrum a large broad band appears centered at $3446 \mathrm{~cm}^{-1}$. This is possibly due to the hydration of PEO. It was experienced that the PEO is highly hydrophilic, thus it absorbs water vapor and gets hydrated. Pure PEO shows a large, broad band of $\mathrm{CH}_{2}$ stretching between 2950 and $2840 \mathrm{~cm}^{-1}$. However, the band is split into two at 2918 $\mathrm{cm}^{-1}$ and $2884 \mathrm{~cm}^{-1}$ corresponding to asymmetric $\mathrm{CH}_{2}$ stretching $\left(\mathrm{v}\left(\mathrm{CH}_{2}\right)_{\mathrm{a}}\right)$ and symmetric $\mathrm{CH}_{2}$ stretching $\left(\mathrm{v}\left(\mathrm{CH}_{2}\right)_{\mathrm{s}}\right)$, respectively. In the intercalated MMTPEO these bands show lower intensity. Most significant changes were observed between 1700 and $800 \mathrm{~cm}^{-1}$. Two clear $\mathrm{CH}_{2}$ vibrational modes appear in PEO at $1464 \mathrm{~cm}^{-1}$ which, correspond to asymmetric $\mathrm{CH}_{2}$ bending $\left(\delta\left(\mathrm{CH}_{2}\right)_{\mathrm{a}}\right)$ and $1358 \mathrm{~cm}^{-1}$ which, corresponds to symmetric $\mathrm{CH}_{2}$ wagging and some $\mathrm{C}-\mathrm{C}$ stretching $\left(\mathrm{w}\left(\mathrm{CH}_{2}\right)_{\mathrm{s}}+\mathrm{v}(\mathrm{CC})\right)$ [27]. Thus in MMTPEO hybrid these bands seem almost absent. This phenomenon is due to the ion dipole interactions between the ether-oxygen of oxyethylene groups and the interlayer cations [25]. This was further confirmed as the intensity of the band of $\left(\mathrm{v}(\mathrm{C}-\mathrm{O})\right.$ at $1640 \mathrm{~cm}^{-1}$ in pure PEO shifted to lower frequency in MMTPEO and the band of $\left(v(\mathrm{O}-\mathrm{H})\right.$ at $1636 \mathrm{~cm}^{-1}$ in MMT is intensed. It is understood that PEO interacts not only with free $-\mathrm{OH}$ of clay layers but with the interlayer cations in a similar manner to conventional PEO-salt complexes. The two bands at $1295 \mathrm{~cm}^{-1}$ and $1252 \mathrm{~cm}^{-1}$ which are related to twisting vibration $\mathrm{t}\left(\mathrm{CH}_{2}\right)_{\mathrm{a}}$ and stretching vibration $\mathrm{v}(\mathrm{C}-\mathrm{O}-\mathrm{C})_{\mathrm{a}}$ [28] are completely absent in MMTPEO. It is worth noted here that a clear band at $1100 \mathrm{~cm}^{-1}$ that corresponds to the $v(\mathrm{C}-\mathrm{O}-\mathrm{C})_{\mathrm{a}}$ vibration mode in PEO is not to be observed in the hybrid MMTPEO. Instead, a broad distorted band 
centered at $1068 \mathrm{~cm}^{-1}$ in MMTPEO was observed. It suggests that significant interactions take place between oxygen and interlayer cations in MMT. The $\mathrm{CH}_{2}$ rocking modes observed in the ranges of $1000-700 \mathrm{~cm}^{-1}$ are sensitive vibrational modes in the conformational arrangements for pure PEO.

To further support the above observation it is clear that the typical modes of $\mathrm{r}\left(\mathrm{CH}_{2}\right)_{\mathrm{s}}$ appear at $949 \mathrm{~cm}^{-1}$ and $844 \mathrm{~cm}^{-1}$ also absent in PEO intercalated MMT. It has been reported in a previous study that the presence of two bands at $949 \mathrm{~cm}^{-1}$ and $844 \mathrm{~cm}^{-1}$ were the characteristic peaks in supporting the gauche conformations of $-\mathrm{CH}_{2}-\mathrm{CH}_{2}$ - groups, as needed for a helical conformation of pure PEO. In addition to that relative lowering of intensities of $920 \mathrm{~cm}^{-1} \mathrm{v}(\mathrm{Al}-\mathrm{O}-\mathrm{H}), 796 \mathrm{~cm}^{-1} \mathrm{v}(\mathrm{Al}(\mathrm{Mg})-\mathrm{O}-\mathrm{H}), 1040-1120 \mathrm{~cm}^{-}$ ${ }^{1} \mathrm{v}(\mathrm{Si}-\mathrm{O})$ and the bands appear at $525 \mathrm{~cm}^{-1}$ and $470 \mathrm{~cm}^{-1}$ correspond to the $v(\mathrm{Al}(\mathrm{Mg})-\mathrm{O}-\mathrm{Si})$ also concede this observation. The overall results confirmed that the crystallinity of PEO has been enormously distorted as the strong interactions with MMT layers and interlayer cations.

The intercalation of PEO within MMTLi showed significant changes in bonding mechanism of the hybrids of MMTLiPEO. It is apparent that intercalated PEO segments tend to interact with $\mathrm{Li}^{+}$cations those are coordinated to Al-O- units in the clay layers favorably. As a consequence of this, the amount of free $\mathrm{O}-\mathrm{H}$ increases as the extent of hydrogen bonded $\mathrm{O}-\mathrm{H}$ decreases. The appearance of the band at $3624 \mathrm{~cm}^{-1} \mathrm{v}(\mathrm{OH})$ stretching mode in MMTLiPEO confirms this assumption. As discussed earlier the typical vibrational modes observed in pure PEO have not been observed in the MMTLiPEO nanocomposite. It is an evident that the PEO also interacts with the relative bonding sites of MMT layers in addition to interlayer cations or $\mathrm{Li}^{+}$cations However, this can be assumed with results of the relative intensity changes of typical vibrational modes in both pure PEO molecules and MMT. The FTIR results observed for the above nanocomposite systems are consistent with results of XRD, and conductivity discussed in earlier sections

\section{CONCLUSIONS}

Montmorillonite (MMT) has been a very promising layered silicate material in the fabrication of nanocomposites. Among many applications of MMT, its use as a conducting material is to be well understood further. The modified MMT showed strategic structure-property relationships as opposed to its unmodified state. It was found that the most easy and simple way to modify the structure of MMT is by intercalation of various cations or molecules within the interlayer spacing. The unique properties of MMT such as self-assembling associate with the ease of production through simple processes make it a very attractive material to be used in electrochemical and electronic devices.

The ionic conductivity observed for the systems of MMT, MMTLi, MMTLiPEO and MMTPEO is rarely enough for practical uses. However, the $\mathrm{Li}^{+}$cations intercalated MMT showed an enhanced ionic conductivity of $5.42 \times 10^{-6} \mathrm{~S} \mathrm{~cm}^{-1}$ at room temperature than that of pure MMT, MMTPEO and MMTLiPEO. The XRD results revealed that the MMTLi is hydrophobic in nature in contrast to other systems. The MMTPEO and MMTLiPEO showed an expanded d spacing of $\sim 18.73 \AA$ at room temperature and with moisturing it increase to $\sim 23.33 \AA$. The MMT shows an ability to reduce the crystalline nature of PEO. The XRD spectra obtained for the systems of MMT and MMTPEO clearly showed this phenomenon. 
The FTIR analysis of the each of above systems showed typical bonding mechanism in the intercalation of $\mathrm{Li}^{+}$cations and PEO within the interlay spacing of MMT, which is in agreement with the corresponding ionic conductivity enhancement. In addition to that it has shown that the crystalline nature of PEO is distorted. Thus it can be suggested that if the structure of the MMT is modified in an appropriate way, significantly enhanced electrical properties can be obtained. Therefore, it is worth pointing out that, a much better understanding of the migration mechanism, transport properties of interlayer cations, the structure of the hybrids and the effect of environmental conditions of each type of systems is a must in order to improve the ionic conductivity in these materials.

\section{References}

1. H. W. Chen, C.Y. Chiu, H.D. Wu, I.W. Shen, F.C. Chang, Polymer. 43(2002)5011

2. P. Lobitz, H. Fullbier, A. Reich, K. Ambrahsat, Solid State Ionics, 58(1992)49

3. M. Alexandre, P. Dubois, Materials Science and Engineering. 28(2000)1

4. F. Croce, G.B. Appetecchi, L. Persi, B. Scrosati, Nature. 394(1998)456

5. J. Liu, J. Pan, J. Chen, Solid State Ionics. 82(1995)225

6. Y. Wenhai, W. Dazhi, Z. Bin, W. Shenjun, X. Lixin, Solid State Commun. 61(1987)271

7. R.L. Goswamee, A. Ayral, K.G. Bhattacharyya, D.K. Dutta, Mater. Lett 46(2-3)(2000)105; Chem. Abstr. 2001, 134 (7), 91602.

8. B. Hamdi, Z. Kessaissia, J.B. Donnet, T.K. Wang, Ann. Chim. (paris) 25(6)(2000)481; Chem. Abstr. 2001, 134 (7), 91510.

9. A. Robledo, N.J. Garcia, J.C. Bazan, Solid State Ionics. 139(2001)303

10. W.A. Deer, R.A. Howie, J. Zussman (Ed.) An introduction to the Rock-Forming Minerals. Essex: ELBS Press, 1992.

11. S.S. Sekhon, S. Gurjinder, S.A. Agnihotry, S. Chandrac, Solid State Ionics. 80(1-2)(1995)37

12. S. Rossi, P.F. Luckham, T.F. Tadros, Colloids and Surfaces A: Physicochem. Eng. Aspects 00. 2002, 1-10.

13. G.O. Barbara, Applied Clay Science. 22(5)(2003)251

14. R.H. Eduardo, J.C. Galvan, J. Merino, B. Casal, P. Aranda, J.M. Antonia, J. M., Solid State Ionics. 85(1-4)(1996)313

15. E. Montarges, L.J. Michot, P. Ildefonse, Microporous and Mesoporous Materials. 28(1999)83

16. E.G. Ralph, Clay Mineralogy. Australia: McGraw-Hill International Series, 1968.

17. M.M. Duane, C.R. Robert, X-Ray Diffraction and the Identification and Analysis of Clay Minerals. Oxford: Oxford University Press, 1989.

18. M.S.Whittingham, Solid State Ionics. 25(1987)295

19. M.S. Whittingham, Solid State Ionics. 32-33(1989)344

20. A. Zecchina, S. Bordiga, G. Spoto, L. Marchese, G. Petrini, G. Leofanti, M. Padovan, J. Phys. Chem. 96(1992)4991

21. J.B. Helen, A.T.R. Simon, M.C. Simon, Mineralogical Magazine, 6(1998)647

22. Z. Bin, X. Lixin, W. Dazhi, Y. Wenhai, Chin, J. Inorg. Mater. 2(1987)176

23. Y. Wenhai, W. Dazhi, Z. Bin, W. Shenjun, X. Lixin, Solid State Commun. 61(1987)271

24. F.M. Gray, Solid Polymer Electrolytes, Fundamentals and Technological Applications. Scotland: VCH Publishers Inc, 1991.

25. J.E. Mark, Polym. Eng. Sci. 36(1996)2905

26. Y. Fan, H. Wu, Solid State Ionics. 93(1997)347 
27. P.A.R.D. Jayathilaka, M.A.K.L. Dissanayake, I. Albinsson, B.E. Mellander, Electrochimica Acta. 47(2002)3257

28. R.M. Silverstein, G.C. Bassler, (Ed.) Spectrometric Identification of Organic Compounds. New York: John Wily and Sons Inc, 1964

(C) 2006 by ESG (www.electrochemsci.org) 